\title{
Dos métodos de anticoagulación en técnicas continuas de depuración extrarrenal
}

\author{
Sergi Aragó Sorrosal ${ }^{1}$, Lida María Rodas Marín², Ferrán Torres³, Vanesa Villegas Jiménez¹, Esteban Poch \\ López de Briñas ${ }^{4}$ \\ ${ }^{1}$ DUE. Sección hemodiálisis. Instituto clínico de Nefrología y Urología. Hospital Clínic de Barcelona. España \\ ${ }^{2}$ Nefróloga. Instituto clínico de Nefrología y Urología. Hospital Clínic de Barcelona. España \\ ${ }^{3}$ Instituto de Investigaciones Biomédicas August Pi i Sunyer (IDIBAPS). Plataforma de bioestadística \\ ${ }^{4}$ Jefe de servicio. Instituto clínico de Nefrología y Urología. Hospital Clínic de Barcelona. España
}

\section{Resumen}

Introducción: Las técnicas continuas de depuración extrarrenal son la terapia de elección en pacientes críticos. Pero, no están exentas de complicaciones como la coagulación del circuito extracorpóreo. El fármaco usado tradicionalmente para la anticoagulación, es la heparina sódica. Aunque también son empleados otros métodos de anticoagulación, como el citrato.

Objetivo: Evaluar la eficacia y seguridad de dos métodos de anticoagulación en pacientes tratados con terapias continuas, en unidades de cuidados intensivos del Hospital Clínic.

Material y Método: Estudio observacional, retrospectivo de 54 sujetos. Se aplicaron los criterios de Insuficiencia renal aguda, descritos en el protocolo "Guía para el tratamiento sustitutivo renal en la insuficiencia renal aguda del Hospital Clínic". Tratados mediante hemodiafiltración, combinando terapias de convección y difusión. Con fracción de filtración $<25 \%$, y dosis de efluente de $30 \mathrm{ml} / \mathrm{kg} / \mathrm{h}$. 27 sujetos realizaron hemodiafiltración, reposición pre-filtro y anticoagulación con heparina sódica. El resto, hemodiafiltración, reposición post-filtro y anticoagulación con citrato. Se valoraron episodios de sangrado y duración en horas de los circuitos extracorpóreos, durante 72 horas de terapia.

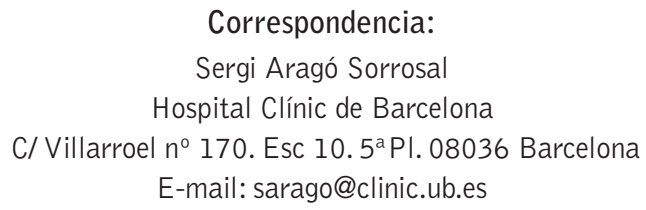

Resultados: El citrato demostró una mayor supervivencia de filtros (95\% IC, MD 65, 44.00-72.00 vs MD $36.00,15.00-22.00, p=0,02$ ). Los pacientes tratados con heparina presentaron más episodios de sangrado, sin diferencias estadísticamente significativas (95\% IC, $n=6$ vs $n=9, p=0.537$ ). Hubo un episodio de hipocalcemia en el grupo citrato, corregido según protocolo. En el grupo heparina, no se presentaron casos de trombocitopenia inducida por heparina.

Conclusiones: El presente estudio, demuestra una mayor eficacia en la supervivencia de los filtros en el grupo citrato.

PALABRAS CLAVE: citrato; heparina; anticoagulación regional; terapia continua depuración extrarrenal; fracaso renal agudo.

\section{Two anticoagulation methods in continuous extra-renal depuration techniques}

\section{Abstract}

Introduction: Continuous extra-renal depuration techniques are the therapy of choice in critically ill patients. But, they are not exempt from complications such as the coagulation of the extracorporeal circuit. The drug traditionally used for anticoagulation is sodium heparin. Although other methods of anticoagulation, such as citrate, are also used.

Objective: To evaluate the efficacy and safety of two anticoagulation methods in patients treated with continuous therapies, in intensive care units of the Hospital Clinic of Barcelona. 
Material and Method: A retrospective observational study with 54 subjects was carried out. The criteria of acute renal insufficiency, described in the protocol "Guide for renal replacement therapy in acute renal failure at the Hospital Clínic" were applied. Patients were treated by hemodiafiltration, combining convection and diffusion therapies; with filtration fraction $<25 \%$, and effluent dose of $30 \mathrm{ml} / \mathrm{kg} / \mathrm{h}$. Twentyseven subjects performed hemodiafiltration, pre-filter replacement and anticoagulation with sodium heparin. The rest, hemodiafiltration, post-filter replacement and anticoagulation with citrate. Episodes of bleeding and duration in hours of the extracorporeal circuits were evaluated during 72 hours of therapy.

Results: Citrate demonstrated a greater survival of filters ( $95 \%$ CI, MD 65, 44.00-72.00 vs. MD 36.00, $15.00-22.00, p=0.02$ ). Patients treated with heparin had more episodes of bleeding, without statistically significant differences $(95 \% \quad C I, n=6$ vs. $n=9$, $\mathrm{p}=0.537$ ). There was an episode of hypocalcemia in the citrate group, corrected according to protocol. In the heparin group, there were no cases of heparin-induced thrombocytopenia.

Conclusions: The present study demonstrates greater efficacy in the survival of filters in the citrate group.

KEYWORDS: citrate; heparin; regional anticoagulation; Continuous therapy; Extra-renal depuration; Acute renal failure.

\section{Introducción}

El fracaso renal agudo (FRA) puede llegar a afectar hasta un $50 \%$ de los pacientes ingresados en unidades de cuidados intensivos (UCI), dato que variará en función del estudio consultado ${ }^{1-3}$. La mortalidad del paciente crítico puede aumentar entre un $35-53 \%$, si presenta FRA asociado al síndrome de disfunción multiorgánica, factor indicador de la complejidad de su situación ${ }^{4}$.

De los pacientes afectados por FRA, entre un 4-10\% precisarán terapias continuas de depuración extrarrenal (TCDE), en alguna de sus modalidades. De los que reciban el alta, hasta un $20 \%$ entrarán en programa de hemodiálisis crónica ${ }^{4-5}$.

Las TCDE son la terapia sustitutiva renal de elección en pacientes que se encuentran en situación crítica, ya que presentan una mejor tolerancia hemodinámica a las mis- mas, a pesar de que la supervivencia no sea mayor que en la hemodiálisis intermitente ${ }^{6-8}$.

Las primeras experiencias en TCDE, aplicadas con hemofiltración arteriovenosa continua, fueron descritas por Kramer en $1977^{\circ}$. Pero desde entonces, muchos han sido los avances introducidos en la terapia. Éstos abarcan tantos aspectos técnicos, introducidos en los monitores, como aspectos de seguridad y eficacia en el tratamiento.

Los cuidados de Enfermería a cargo de pacientes tratados con TCDE, incluyen los cuidados habituales de un paciente crítico, e integran los cuidados de la terapia y del acceso vascular (AV). Tales como mantener el flujo de sangre adecuado (Qb), la permeabilidad del circuito extracorpóreo, flujos de terapia, monitorización de las presiones del circuito, e indicación de la anticoagulación. Referentes al AV, habitualmente catéter temporal, mantener la permeabilidad y el correcto funcionamiento $0^{2-3,10}$.

Entre las complicaciones de las TCDE relacionadas con la técnica, destacan: la disfunción del AV, y la coagulación del circuito extracorpóreo $0^{2-3,10-11}$. Continúan siendo uno de los puntos débiles de la terapia, y los cuidados de Enfermería irán encaminados a minimizarlos. Para la anticoagulación del circuito, el fármaco utilizado tradicionalmente ha sido la heparina sódica (HS); anticoagulando de forma sistémica al paciente, con el riesgo de sangrado y trombocitopenia inducida (TIH) que puede suponer, para aquéllos que se encuentran en situación crítica $^{12}$.

Debido a estas complicaciones, otros métodos de anticoagulación empezaron a utilizarse en las TCDE. La heparina en diferentes modalidades (regional, no fraccionada o de bajo peso molecular), prostaciclinas (epoprostenol), o inhibidores directos de la trombina (argatrobán) son algunos ejemplos ${ }^{2,11,13-14}$.

El citrato, cuyo efecto es regional, actúa sólo a nivel del circuito extracorpóreo; evitando la anticoagulación del paciente. La primera experiencia con citrato fue descrita por Morita et al en 1961, en pacientes en hemodiálisis con riesgo de sangrado ${ }^{15}$. Pero no fue hasta los años 80 , cuando aparecieron más estudios sobre su uso en hemodiálisis. Se introdujo en pacientes críticos y TCDE en la década de los $90^{16-18}$.

La acción del citrato consiste en quelar el calcio ionizado del paciente, que actúa en varios niveles de la cascada de coagulación, evitando la activación de la 
misma, inhibiendo la formación de fibrina y la agregación plaquetaria. En las TCDE, el citrato se administra a través de la línea aferente del circuito extracorpóreo; evitando la coagulación del mismo de forma regional, sin anticoagular al paciente, neutralizándose su efecto de dos maneras diferentes: un porcentaje de citrato (que puede variar desde el 30 al $60 \%$ en función de los estudios) es eliminado a través del hemofiltro; mientras que la posible carga de citrato que se infunde al paciente, dado que éste posee efecto buffer, es metabolizado a través de hígado, músculo y corteza renal, dando lugar a bicarbonato sódico (generándose tres moles de bicarbonato por cada mol de citrato, y liberando el calcio ionizado) $)^{19-21}$.

Durante el tratamiento de TCDE con citrato, se debe reponer el calcio del paciente, para compensar la pérdida del mismo que se produce al quelarse junto al citrato en el circuito extracorpóreo, y ser eliminado éste último en parte a través del hemofiltro. La reposición de calcio siempre se realizará post-filtro.

De lo descrito, se deriva que la administración de citrato puede presentar complicaciones, entre las que destacan $^{22-23}$ :

- Episodios de hipernatremia: dado que el citrato utilizado para las TCDE es citrato trisódico $\left(\mathrm{Na}_{3} \mathrm{C}_{6} \mathrm{H}_{5} \mathrm{O}_{7}\right)$.

- Episodios de hipomagnesemia: ya que el citrato también forma complejos con el magnesio, y ambos son eliminados en el hemofiltro.

- Alteraciones del estado ácido-base metabólico: alcalosis metabólica, por acúmulo de citrato durante el tratamiento y debido a su efecto buffer. Por ello, se evita el uso de citrato en pacientes con insuficiencia hepática.

- Episodios de hipocalcemia: ya que el citrato unido al calcio en el circuito extracorpóreo, es eliminado a través del filtro, dando lugar a la disminución de calcio sérico del paciente.

Por tanto, el objetivo de este estudio es evaluar la eficacia y seguridad de dos métodos de anticoagulación utilizados en pacientes tratados con TCDE, en unidades de cuidados intensivos (UCIs) del Hospital Clínic de Barcelona.

\section{Material y Método}

Estudio observacional, prospectivo, en una población de pacientes con TCDE ingresados en UCIs del Hospital Clínic de Barcelona, durante los meses de enero a septiem- bre de 2016. Fueron incluidos 54 sujetos ingresados en las UCIs de cirugía cardiovascular, coronaria y quirúrgica. El muestreo realizado fue no probabilístico intencional.

Como criterios de inclusión se establecieron: TCDE superior a 72 horas de duración, portador de AV temporal, sin antecedentes de TIH. Se excluyeron a aquellos pacientes con contraindicación para la anticoagulación, insuficiencia hepática, IRC en estadio V en programa de hemodiálisis crónica.

Los criterios de insuficiencia renal aguda (IRA) aplicados, fueron los descritos en el protocolo "Guía para el tratamiento sustitutivo renal en la insuficiencia renal aguda del Hospital Clínic".

Respecto a la TCDE, la modalidad de tratamiento fue la hemodiafiltración (HDFVVC), que combina terapias convectivas y de difusión, con una fracción de filtración que no superaba el $25 \%$ ( $\%$ de agua plasmática que es extraída de la sangre en función del volumen que entra en el filtro, a través de su membrana); y una dosis de efluente de $30 \mathrm{ml} / \mathrm{kg} / \mathrm{h}$.

Se trataron 27 sujetos con anticoagulación sistémica con HS (ASHS), y HDFVVC con reposición pre-filtro. $Y$ el resto, con anticoagulación regional con citrato (ARC), y HDFVVC con reposición post-filtro. El estudio incluyó un seguimiento de 72 horas para cada paciente. El monitor utilizado para la terapia fue el Multifiltrate ${ }^{\circledR}$, con versión 5.2 de software. La membrana de los hemofiltros fue polisulfona con superficie de $1.8 \mathrm{~m}^{2}$. Se empleó un equipo de TCDE con líneas incorporadas para la infusión de citrato y calcio, en el grupo ARC.

En la tabla 1, se detallan características diferenciales de la TCDE de ambos grupos.

Como criterios de interrupción de la TCDE con citrato, se utilizaron los siguientes:

n Cociente de calcio total/calcio ionizado > $2.5 \mathrm{mmol} / \mathrm{l}$. - Alcalosis metabólica persistente con exceso de base (EB) > $10 \mathrm{mmol} / \mathrm{l}$.

- Signos clínicos de hipocalcemia.

- Acidosis metabólica progresiva.

Las variables estudiadas incluyeron las características demográficas de los pacientes, y variables relacionadas con la seguridad y eficacia de las TCDE. Las variables descritas para seguridad fueron episodios de sangrado, soporte transfusional, y episodios de TIH e hipocalcemia. En cuanto a eficacia, duración en horas de los 
Tabla 1. Características diferenciales de las terapias continuas de depuración extrarrenal de ambos grupos de anticoagulación.

\begin{tabular}{lll}
\hline & Grupo ASHS & Grupo ARC \\
\hline Qb & $150-200 \mathrm{ml} / \mathrm{min}$ & En función del peso del paciente \\
\hline Qd y Qr & en función del peso y la dosis de efluente & En función del peso y la dosis de efluente \\
\hline Anticoagulante & HS-dilución: $1 \mathrm{ml}=2,5 \mathrm{mgr}(250 \mathrm{UI})$ a $1-2 \mathrm{ml} / \mathrm{h}$ & $\begin{array}{l}\left.\text { Citrato al } 4 \% \text { (Kabi Bag }{ }^{\circledast} 136 \mathrm{mmol} / \mathrm{l}\right) \\
\text { Dosis inicial: } 5 \mathrm{mmol} / \mathrm{l}\end{array}$ \\
\hline UF & Máximo $100 \mathrm{ml} / \mathrm{h}$ & Máximo $100 \mathrm{ml} / \mathrm{h}$
\end{tabular}

$Q_{b}$ : Flujo de sangre.

$Q_{d}$ y $Q_{r}$ : Flujo de diálisis y de reposición (mil/h).

UF: Ultrafiltración.

Anticoagulante $\boldsymbol{A R C}$ : La dosis de citrato se modificó según el calcio iónico post-filtro, muestra de la línea eferente del circuito, manteniéndose en 0.25-0.34 mmol/l.

ASHS: El líquido de diálisis y el de reposición fue el mismo, Multibic ${ }^{\circledR} \mathrm{K} 3$.

ARC: Líquido de diálisis usado fue $\mathrm{Ci}-\mathrm{Ca}{ }^{\circledR} \mathrm{K} 2$, y de reposición, MultiBic ${ }^{\circledR} \mathrm{K} 3$. La reposición se realizó post-filtro, para evitar la interacción del calcio contenido en el líquido de sustitución, con el citrato.

Solución cloruro cálcico $\boldsymbol{A R C}$ : Infundida al final del circuito extracorpóreo o por vía central no conectada al equipo de TCDE.

Se modificó a partir del calcio sérico del paciente, para mantener una concentración de 1,12-1,20 mmol/l.

equipos extracorpóreos, y número de los mismos utilizados en cada grupo.

Las variables categóricas se han descrito con frecuencias y porcentajes, las continuas con medias y desviación estándar o mediana y amplitud intercuartil (percentil 25-percentil 75) de acuerdo a su distribución. La estrategia general de análisis se estableció de la siguiente manera: para comparar entre variables categóricas se utilizó la prueba exacta de Fisher entre grupos, para las continuas la t de student y en caso de no cumplirse las asunciones de aplicabilidad, se aplicaron métodos no paramétricos (prueba de U de Mann-Whitney). Las variables de evaluación repetida a lo largo del tiempo como EB o Calcio, se analizaron usando modelos mixtos para medidas repetidas.

Se consideró el valor de $p<0,05$ como estadísticamente significativo, y el análisis se realizó con la versión 9.4 de software del paquete estadístico SAS (SAS Institute Inc, Cary, NC).

El estudio fue aprobado por el Comité de Ética e Investigación del Hospital Clínic.

\section{Resultados}

Un 70,4\% (38) eran hombres, con una media de edad de $70.19 \pm 11.79$ años, un peso medio de $73.25 \pm 15.60$ kilogramos; y una media de índice de comorbilidad de Charlson de $7.56 \pm 1.31$ puntos.

Para conocer el estado crítico de los pacientes, se aplicaron escalas de gravedad. Como Acute Physiology and Chronic Health Evolution Score II (APACHE II), con una media de $24.06 \pm 5.89$ puntos; y Sepsis-related organ failure assessment (SOFA), con una media de $11.28 \pm 2.7$ puntos. Todos los pacientes precisaron soporte vasoactivo, y un $72.2 \%$ (39) estuvieron ventilados mecánicamente durante la terapia.

Las características demográficas y epidemiológicas de los pacientes estudiados $(n=54)$, por grupo de anticoagulación se pueden observar en la tabla 2.

Un $51.9 \%$ (28) de los pacientes presentaban algún estadio de insuficiencia renal crónica (IRC), sin requerimiento dialítico previo. 
Todos eran portadores de AV temporal. La mayoría de ellos localizados en vena femoral $88.9 \%$ (48), yugular derecha $7.4 \%$ (4), y yugular izquierda $3.7 \%(2)$.

Durante el tiempo de estudio, las 54 terapias comprendieron un total de 109 circuitos extracorpóreos: 43 circuitos pertenecieron al grupo de ARC; y los 66 restantes, a los pacientes tratados con ASHS.

Durante las 72 horas de seguimiento de los pacientes, se requirió un mayor número de circuitos en las HDFVVC con ASHS, que en las HDFVVC con ARC (ASHS=2.44 \pm 1.15 circuitos vs $A R C=1.59 \pm 0.75$ circuitos, $p=0.002$ ).

En cuanto a eficacia, los circuitos con ARC presentaron una mayor supervivencia en horas, que el grupo de ASHS (95\% IC, MD 65.00, 44.0072.00 horas vs MD 36.00, 15.00-72.00 horas, $p=0.02)$, figura 1 . A pesar de que un total de $9(33.3 \%$ ) circuitos del grupo de ARC fueron sustituidos por coagulación, comparados con los 17 (63\%) del grupo de ASHS ( $p=0.027$ ); destacar que 3 ( $11.1 \%$ ) circuitos del grupo de ARC se coagularon por disfunción del $\mathrm{AV}$, y sólo 1 (3.7\%) por este motivo, en el grupo de ASHS ( $p=0.027)$.

Un mayor número de circuitos fueron cambiados de forma electiva, Ilegando a las 72 horas de funcionamiento (tiempo recomendado como máximo de uso sin pérdida de eficacia), en la ARC vs ASHS (95\% IC, $n=9$ $(33.3 \%)$ vs $n=1(3.7 \%), p=0.027)$.

Relacionado con la seguridad, los pacientes tratados con ARC presentaron menos episodios de sangrado activo que el grupo de ASHS, pero no hubo diferencias estadísticamente significativas ( $95 \%$ IC, $n=6(22.2 \%)$ vs $n=9(33.3 \%), p=0.537)$. El número de pacientes que requirieron soporte transfusional fue similar en ambos grupos (95\% IC, $n=19(70.3 \%)$ vs $n=18(66.6 \%)$, $p=0.985$ ).

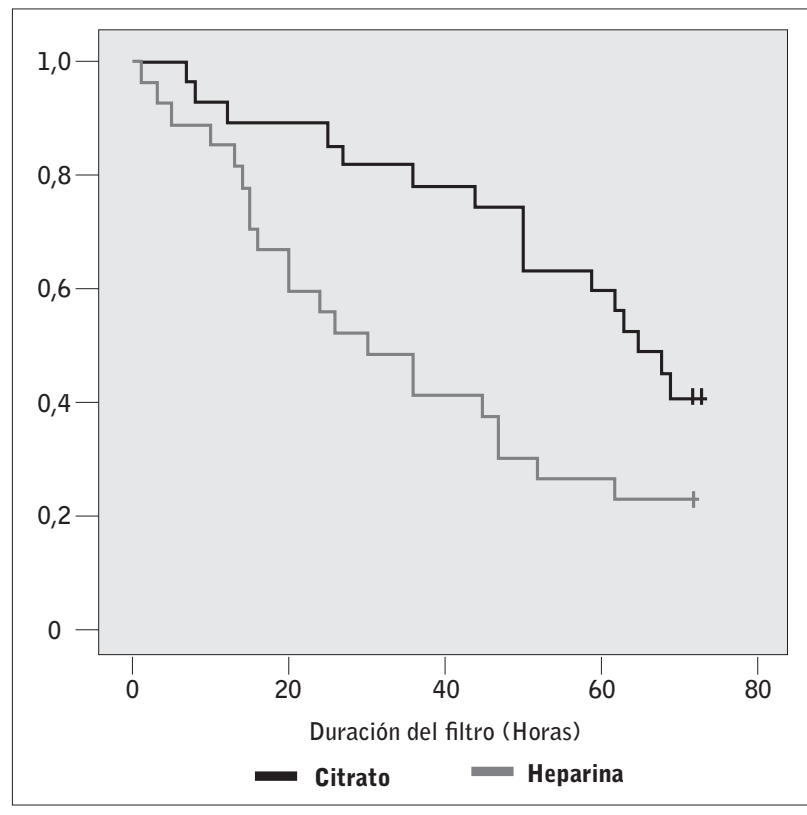

Figura 1. Curva de Kaplan-Meier para la supervivencia de los circuitos extracorpóreos (en horas) en función de la anticoagulación utilizada. 
Cabe destacar que a estadio más avanzado de IRC previo, el requerimiento transfusional fue mayor ( $95 \%$ IC, estadio $3=0.91 \pm 1.38$ vs estadio $4=2.59 \pm 2.12, p=0.045$ ).

En cuanto al grupo de ASHS, no se dio ningún caso de TIH. Y en el grupo de ARC, se presentó un solo caso de hipocalcemia, que fue corregido según protocolo. La evolución de los niveles de Calcio ionizado ( $\mathrm{Ca} 2+)$, y Calcio total en el grupo de ARC se pueden observar en las figuras 2 y 3 . No se objetivaron alteraciones metabólicas del equilibrio ácido-base significativas. Se produjo un aumento del exceso de base en el grupo de pacientes con ARC, sin exceder los límites de la normalidad, por la propia corrección de la IRA. EI EB sólo se estudió en el grupo ARC (ver figura 4).

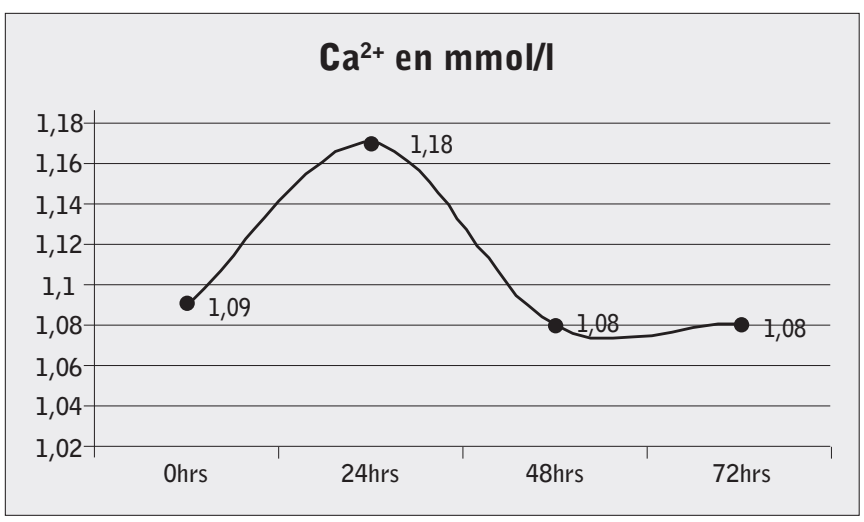

Figura 2. Valores de Calcio iónico $\left(\mathrm{Ca}^{2+}\right)$, durante el período de estudio en el grupo ARC.

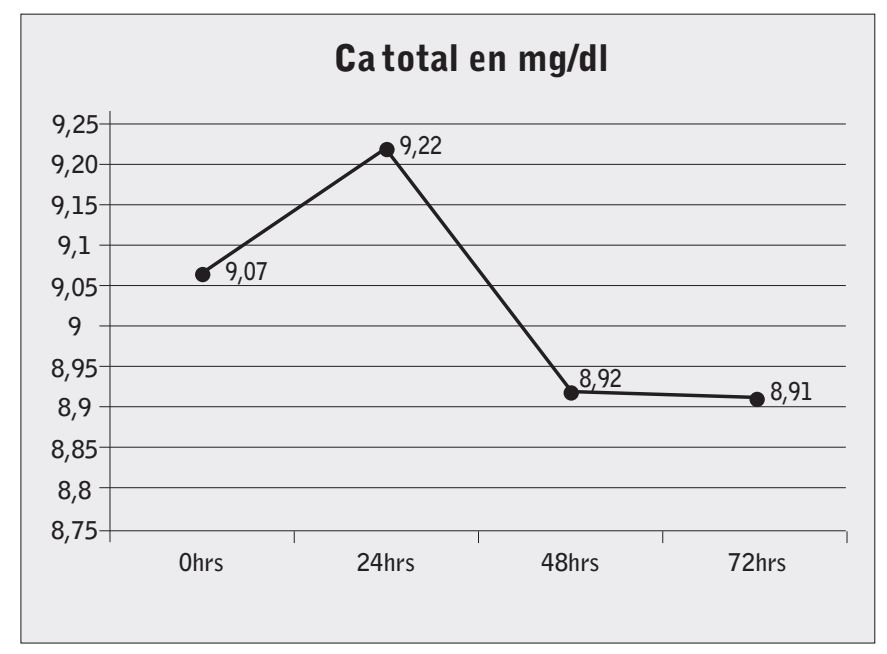

Figura 3. Valores de Calcio total $\left(\mathrm{Ca}^{+}\right.$total), durante el período de estudio en el grupo ARC.

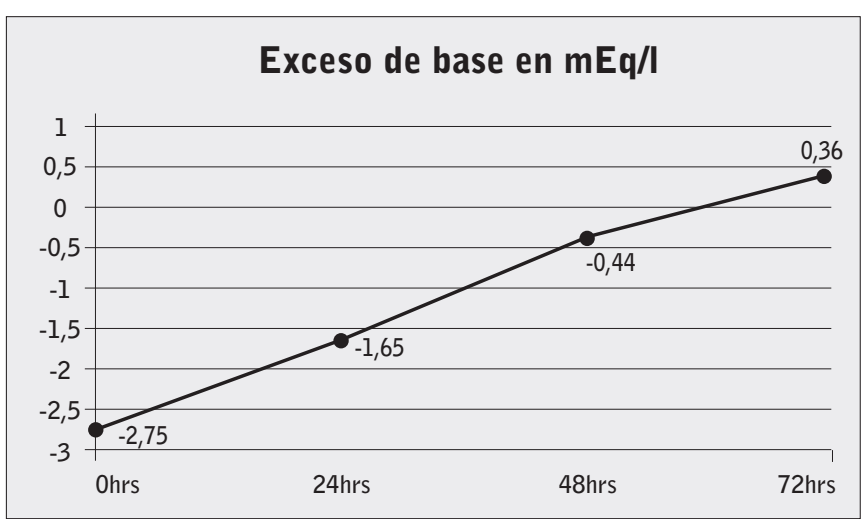

Figura 4. Valores de Exceso de base, durante el período de estudio en el grupo ARC.

En cuanto a la dosis de citrato y calcio administradas, expresadas en medias y desviación estándar, en $\mathrm{mmol} / \mathrm{l}$ : Citrato $=4.60 \pm 0.58$ y Calcio $=1.94 \pm 0.21$.

La mortalidad de los pacientes del grupo estudiado fue del $35.2 \%$, una vez finalizado el estudio (ARC $n=8$ $(29.6 \%)$, ASHS $n=11(40.7 \%))$.

\section{Discusión / Conclusiones}

El objetivo del presente trabajo ha sido comparar dos métodos de anticoagulación: HS administrada de forma sistémica; y citrato, actuando a nivel regional. Siendo el primer estudio realizado en el Hospital Clínic sobre ARC.

Aunque con una muestra de estudio pequeña, comparte resultados con otros estudios más extensos. Éstos han comparado la eficacia de la ARC y ASHS con diferentes métodos. Usando terapias de convección, hemofiltración, convección y difusión, HDFVVC; con reposición de líquidos post-filtro o pre-filtro y con ASHS, o incluso de forma regional (administración de HS junto a protamina-ARH), de amplio uso en Australia y Nueva Zelanda ${ }^{13-14,24-25}$. Los estudios muestran mejor eficacia de la ARC, para mantener la permeabilidad del circuito extracorpóreo, evitar su coagulación y la aparición de $\mathrm{TIH}^{13,24-25}$.

En nuestro estudio, hemos comparado AHS infundiendo el líquido de reposición pre-filtro; y ARC con reposición post-filtro. En el grupo AHS, se realizó la reposición del líquido de infusión pre-filtro, para favorecer la permeabilidad del circuito ${ }^{3}$. Se optó por este tipo de reposición porque previene y/o evita la coagulación del circuito en 
comparación con el mismo procedimiento pero con infusión post-filtro; y aunque condiciona el aclaramiento de pequeñas moléculas, como la urea, no existen evidencias de que una mayor eficacia de la diálisis, mejore la supervivencia del paciente crítico ${ }^{26}$. Por tanto, y aun comparando la ARC con infusión post-filtro con la AHS con reposición pre-filtro, que previene la coagulación del circuito, la ARC demostró mayor eficacia en la supervivencia de la técnica.

En cuanto al riesgo de sangrado, el grupo tratado con ARC presentó un menor número de episodios de sangrado activo, aunque sin diferencias significativas; dato que se relaciona con los obtenidos en el resto de trabajos publicados, que favorecen el uso de la $\mathrm{ARC}^{13-14,24-25}$. No ocurre lo mismo en cuanto al requerimiento de concentrados de hematíes que fue similar en ambos grupos, y no coincide con lo presentado hasta el momento; si se comparan ARC con ASHS, o con ARH, se precisan un número menor de concentrados en el grupo $A R C^{13-14,27}$.

Sobre las complicaciones de ambos métodos de anticoagulación, todos los trabajos destacan el riesgo de presentar episodios de hipocalcemia o alcalosis metabólica de los pacientes tratados con ARC; y de sangrado o TIH de los tratados con ASHS. En este estudio, sólo se dió un caso de hipocalcemia, que fue corregido según el protocolo, sin repercusión clínica para el paciente. Y no se presentaron casos de $\mathrm{TIH}^{13-14,24-25}$.

A pesar de que en la mayoría de trabajos, la insuficiencia hepática sea un criterio de exclusión para la ARC; hay dos estudios, que estiman efectiva y viable la ARC, con mayor atención a los posibles cambios metabólicos, en estos pacientes ${ }^{28-29}$.

Algunas de las características demográficas del grupo de estudio se comparten con otros trabajos. Pacientes predominantemente varones con media de 66 años de edad, y la localización de los AVs fue en un porcentaje muy alto en vena femoral ${ }^{5}$, a pesar de las recomendaciones de las KDIG030.

Aun siendo un estudio pequeño, la mortalidad de los pacientes es muy similar a la del resto de trabajos, un $35,2 \%$ de los pacientes. Y ésta, no está relacionada con la TCDE o con el método de anticoagulación utiliza$\mathrm{do}^{4-5,13-14,24-25}$.

El análisis de coste-efectividad, se ha realizado en varios trabajos y precisa de más estudios y evidencia científica; aunque se atribuye un menor coste a la ARC asociado a un menor número de circuitos utilizados, y complicaciones ${ }^{13-14,25,27,31}$.

Varias son las limitaciones, como el hecho de que sea un trabajo observacional sin randomización, de un solo centro, con una población de estudio pequeña, y comprenda un periodo corto de tiempo; provocando que no pueda poseer validez externa. Pero a pesar de ello, está apoyado por resultados similares de otros estudios más extensos.

La ARC presenta una mayor eficacia en la supervivencia de los filtros y evitando la coagulación. El riesgo de sangrado es menor que en la ASHS, y presenta escasas complicaciones.

Agradecimientos: A todas las personas y pacientes que han colaborado en este estudio, y principalmente a los equipos de Enfermería de las unidades de cuidados críticos participantes.

El autor declara que no hay conflicto de interés.

Recibido: 5 diciembre 2017

Revisado: 14 febrero 2018

Modificado: 17 febrero 2018

Aceptado: 20 febrero 2018

\section{Bibliografía}

1. Hoste EA, Clermont G, Kersten A, Venkataraman $\mathrm{R}$, Angus DC, De Bacquer D et al. RIFLE criteria for acute kidney injury are associated with hospital mortality in critically ill patients: a cohort analysis. Crit Care. 2006; 10: R73.

2. Richardson A, Whatmore J. Nursing essential principles: continuous renal replacement therapy. Nurs Crit Care. 2015; 20(1): 8-15.

3. Romero $M$, de la Cueva L, Delgado P. Actualización en técnicas continuas de reemplazo renal. Enferm Int. 2013; 24(3): 113-119.

4. Navas $A$, Ferrer R, Martínez M, Martínez ML, De Haro C, Artigas A. Terapia de reemplazo renal en 
paciente crítico: cambios evolutivos del tratamiento en los últimos años. Med Int. 2012; 36(8): 540547.

5. Tomasa TM, Sabater J, Poch E, Fort J, Lloret MJ, Roca $\mathrm{J}$ et al. Manejo actual de las terapias continuas de remplazo renal: Estudio epidemiológico multicéntrico. Med Int. 2017; 41(4): 216-226.

6. Rabindranth K, Adams J, Macleod AM, Muirhead N. Intermittent versus continuous renal replacement therapy for acute renal failure in adults. Cochrane Database of Syst Rev. 2007; (3): CD003773.

7. Bagshaw SM, Berthiaume LR, Delaney A, Bellomo R. Continuous versus intermittent renal replacement therapy for critically ill patients with acute kidney injury: A meta-analysis. Crit Care Med. 2008; 36(2): 610-617.

8. Ghahramani N, Shadrou S, Hollenbeak C. A systematic review of continuous renal replacement therapy and intermittent haemodialysis in management of patients with acute renal failure. Nephrol. 2008; 13(7): 570-578.

9. Kramer P, Wigger W, Rielger J, Matthaei D, Scheler F. Arteriovenous haemofiltration: a new and simple method for trestment of over-hydrated patients resistant to diuretics. Klin Wochenschr. 1977; 55(22): 1121-1122.

10. Olert A, Hernández AI, Miralles FJ, Cortés J, Domínguez MA, Caro ME. Experiencia con las técnicas continuas de reemplazo renal en cuidados intensivos. Determinantes de la duración del hemofiltro. Rev Soc Esp Enferm Nefrol. 2008; 11(4). 259-264.

11. Molano E, Guirao A, Esteban ME, Sanz P, García E, Sánchez-Izquierdo JA. Detección de riesgos para la seguridad del paciente en la implementación de técnicas continuas de depuración extracorpórea. Enferm Int. 2011; 22(1): 39-45.

12. Syed S, Reilly RF. Heparin-induced thrombocytopenia: a renal perspective. Nat Rev Nephrol. 2009; 5: 501-511.

13. Gattas DJ, Rajbhandari D, Buhr H, Bellomo R. A randomized controlled trial of regional citrate versus regional heparin anticoagulation for continuous renal replacement therapy in critically III adults. Crit Care Med. 2015; 48(8): 1622-1629.

14. Gutiérrez-Bernays D, Ostwald M, Anstey C, Campbell V. Transition from heparin to citrate anticoagulation for continuous renal replacement therapy: safety, efficiency, and cost. Ther Apher Dial. 2016; 20(1): 53-59.

15. Morita Y, Johnson RW, Dorn RE, Hall DS. Regional anticoagulation during hemodialysis using citrate. Am J Med. 1961; 242(1): 32-43.

16. Von Brecht JH, Flanigan MJ, Freeman RM, Lim VS. Regional anticoagulation: hemodialysis with hipertonic trisodium citrate. Am J Kidney Dis. 1986; 8(3): 196-201.

17. Flanigan MJ, Von Brecht JH, Freeman RM, Lim VS. Reducing the hemorrhagic complications of hemodialysis: a controlled comparison of low-dose heparin and citrate anticoagulation. Am J Kidney Dis. 1987; 9(2): 147-153.

18. Bellomo R, Teede H, Boyce N. Anticoagulant regimens in acute continuous hemodiafiltration: a comparative study. Int Med Care. 1993; 19(6):329-332.

19. Calatzis A, Toepfer M, Schramm W, Spannagl M, Schiffl H. Citrate anticoagulation for extracorporeal circuits: effects on whole blood coagulation activation and clot formation. Nephrol. 2001; 89: 233-236.

20. James MF, Roche AM. Dose-response relationship between plasma ionized calcium concentration and thrombelastography. J Cardiothorac Vasc Anesth. 2004; 18(5): 581-586.

21. Carneiro P, Santiago MJ, López J, Castillo A, López-Herce J. Anticoagulación regional con citrato en las técnicas de depuración extrarrenal continuas. An Pediatr. 2012; 76(1): 49-52.

22. Morgera $S$, Scholle $C$, Voss $G$, Haase $M$, Vargas-Hein 0 , Krausch $D$ et al. Metabolic complications during regional citrate anticoagulation in continuous venovenuos hemodiafiltration. Nephrol Clin Pract. 2004; 97(4): 131-136.

23. Fernández J, Oliveros $C P$, González LS, Gastelbondo R, Mulett H, Godoy J et al. Guía de manejo 
anticoagulación con citrato de sodio para terapia de reemplazo renal en niños. Acta Colom Cuid Int. 2012; 12(3): 179-184.

24. Bai M, Zhou M, He L, Ma F, Li Y, Yu Y et al. Citrate versus heparin anticoagulation for continuous renal replacement therapy: an updated meta analysis of RCTs. Int Med Care. 2015; 41(12): 2098-2110.

25. Liu C, Mao Z, Kang H, Hu J, Zhou F. Regional citrate versus heparin anticoagulation for continuous renal replacement therapy in critically ill patients: a meta-analysis with trial sequential analysis of randomized controlled trials. Crit Care. 2016; 20(1): 1-13.

26. The Renal Replacement Therapy Study Investigators, Bellomo R, Cass A, Cole L, Finfer S, Gallagher $M$, et al. Intensity of continuous renal-replacement therapy in critically ill patients. N Engl J Med. 2009; 361(17): 1627-1638.

27. Park JS, Kim GH, Kang CM, Lee $\mathrm{CH}$. Regional anticoagulation with citrate is superior to systemic anticoagulation with heparin in critically patients undergoing continuous venovenous hemodiafiltration. Korean J Intern Med. 2011; 26(1): 68-75.

28. De Vico P, Messino V, Tartaglione A, Beccaris C, Buonomo C, Talarico D et al. Safety and efficacy of citrate anticoagulation continuous renal replacement therapies in post-cardiac surgery patients with liver dysfunction. Ther Apher Dial. 2015; 19(3): $272-278$
29. Schultheiss C, Saugel B, Phillip V, Thies P, Noe S, Mayr $U$ et al. Continuous venovenous hemodialysis with regional citrate anticoagulation in patients with liver failure: a prospective observational study. Crit Care. 2012; 16: R162.

30. KDIGO: clinical practice guideline for the evaluation and management of acute kidney injury. Kidney Int Suppl. 2012; 2:3. Doi:10.1038/ki sup.2012.3

31. Hafner S, Stahl W, Fels T, Träger K, Georgieff M, Wepler M. Implementation of continuous renal replacement therapy with regional citrate anticoagulation on a surgical and trauma intensive care unit: impact on clinical and economic aspects-an observational study. J Int Care. 2015(3): 35-43.

Este artículo se distribuye bajo una Licencia Creative Commons Atribución-NoComercial 4.0 Internacional. https://creativecommons.org/licenses/by-nc/4.0/ 